\title{
Review Article \\ What Makes Oral Candidiasis Recurrent Infection? A Clinical View
}

\author{
Azmi M. G. Darwazeh and Tamer A. Darwazeh \\ Department of Oral Medicine \& Surgery, Faculty of Dentistry, Jordan University of Science \& Technology, \\ P.O. Box 3030, Irbid 22110, Jordan \\ Correspondence should be addressed to Azmi M. G. Darwazeh; darwazeh@just.edu.jo
}

Received 18 September 2014; Accepted 13 December 2014; Published 28 December 2014

Academic Editor: Terezinha Inez Estivalet Svidzinski

Copyright (c) 2014 A. M. G. Darwazeh and T. A. Darwazeh. This is an open access article distributed under the Creative Commons Attribution License, which permits unrestricted use, distribution, and reproduction in any medium, provided the original work is properly cited.

Clinical oral Candida infection (candidiasis) is one of the common oral mucosal infections, and its management is usually frustrating due to either treatment failure or recurrence. Historically, oral candidiasis has been branded as disease of diseased. The unsuccessful management of oral candidiasis can due to either incorrect diagnosis, failure to identify (or correct) the underlying predisposing factor(s), or inaccurate prescription of antifungal agents. Failure to properly treat oral candidiasis will lead to persistence of the fungal cell in the oral cavity and hence recurrence of infection. The oral health care provider should be aware of these fall pits in order to successfully manage oral candidiasis.

\section{Introduction}

Oral candidal colonization and candidiasis have recently received increased attention by the health care providers and researchers alike, particularly following the emergence of human immunodeficiency virus (HIV) infection and the widespread use of broad spectrum antibiotics and immunosuppressant therapy [1]. The genus Candida comprised more than 150 species which are widely spreading in the environment. Knowing that the majority of the species cannot live at the human body temperature [2] explains why the oral cavity is colonized with only a limited number of Candida species.

Candida species constitute part of the oral harmless commensal flora in about $2-70 \%$ of the general population but is responsible for causing infection if the host immune barriers are breached either on the local or on the systemic level [1]. Candida albicans is the species largely responsible for oral candidiasis which is the most common human fungal infection especially in childhood and the elderly. It is not uncommon to encounter a recurrence of the oral candidal infection after some time of institution of antifungal therapy, which constitutes a frustration and disappointment for both the clinician and the patient. One study estimated that around $20 \%$ of patients with oral candidiasis experience infection recurrence and in around 30\% of the recurrences the second isolate was different from that responsible for the first episode of infection [3]. This raises the question whether the "recurrence" is a second infection or due to "persistent" Candida cells.

If the superficial oral candidal infection was not well managed in severe immunosuppression, the patient may become susceptible to esophageal spread of infection or to the potentially lethal systemic candidemia [4]. Therefore, it is essential for the oral candidal infection to be diagnosed accurately and managed appropriately to avoid its recurrence or systemic spread. This paper is discussing the various reasons facilitating the recurrence or treatment failure of oral candidiasis.

\section{Oral Candida "Carriage" versus "Infection"}

The mere isolation of any of the Candida species from the oral cavity of a subject without the presence of clinical signs and symptoms of infection is described as "Candida carriage." The epidemiological studies have shown that limited Candida species were able to colonize the mouth (and other body surfaces such as skin, vagina, and gastrointestinal mucosa) of human being. The commonly isolated species are Candida 
albicans, C. glabrata, C. tropicalis, and C. krusei and to less extent C. lusitaniae, C. dubliniensis, C. kefyr, C. guilliermondii, C. parapsilosis, and C. lipolytica [1]. Only the first three are commonly isolated from oral clinical infections. Candida albicans is the species most encountered in health subjects and infection. The genus Candida is a highly heterogeneous group of yeast-like fungi which markedly differ in their biochemical, morphological, and genetic composition. This explains the differences in their ability to elicit infection [2]. However, it is still unclear why only some individuals become "carriers" and with widely variable number of colonies, despite the fact that Candida species are ubiquitously distributed in nature. Similarly what determines that certain species colonize the oral cavity preferentially is not precisely determined yet.

Candidal adhesion to oral mucosa has been long recognized as the essential step in the process of colonization and infection. This process of adhesion is complex and multifactorial. The fact that both Candida and epithelial cell surface are negatively charged means that there are repulsive forces retarding their adhesion. Nevertheless, there are other attractive forces such as Lifshitz-van der Waals forces, hydrophobic interactions, and Brownian movement forces. The sum of these nonspecific forces will determine whether the initial nonspecific adhesion between fungal and epithelial cells will be established. After the repulsive forces have been overcome, adherence of candidal cells to epithelial cells is then established between specific "adhesions" on the fungal cell surface and "ligands" on the epithelial cell surface [5]. However, the ability of Candida cells to adhere is generally concentration- and species/strain-dependent. In addition some environmental factors, such as saliva and interaction with other microbial flora, may affect this adhesion [6]. The variability of these factors between individuals may partly determine who will become Candida carriers. Once oral Candida adherence to oral mucosa has been established, then colonization and growth are mandatory for the persistence of the organism on the surface. Subsequently, clinical infection will become evident when the integrity of the immune system is breached either on the local or on the systemic level which constitutes risk factors (predisposing factors) for oral candidiasis. Although C. albicans is the causative organism for the majority of oral candidiasis, C. krusei can cause infection in severely immunocompromised patients and C. glabrata in patients receiving radiotherapy [7]. New species, such as C. dubliniensis and C. inconspicua, have been recognized in HIV-infected patients [8].

In clinical practice, two tests are essential to diagnose oral candidiasis. Oral swab obtained from the lesion is usually cultured on the selective medium, for example, Sabouraud's agar, and incubated aerobically for approximately 48 hours. This is combined with oral smear test and direct microscopy following rapid staining. Candida species stain poorly by hematoxylin and eosin; therefore, staining with periodic acid-Schiff (PAS), Gridley stain, or Gomori methenamine silver (GMS) stain is in use [9]. It is widely accepted clinically that combining the presence of the clinical signs suggestive for oral candidiasis and positive results of swab and smear tests is confirmatory for the clinical candidal infection.

\section{Factors Associated with Recurrence or Recalcitrance of Oral Candidiasis}

3.1. Failure to Identify Predisposing Factor(s). The transition of the innocuous commensal Candida to pathogenic organisms may be associated with the virulence attributes of the organism such as that evident in C. albicans [5]. Nevertheless, it is generally accepted that the host factors (Table 1) are of higher critical importance in the development of the disease state. Historically, this was noticed by the ancient Greek physician Hippocrate (460-370 BC) who described oral candidiasis as "disease of diseased."

One study has shown that about $30 \%$ of physicians admitted they would prescribe nystatin for oral thrush in infants on the request of nursing staff without examining the patient's oral cavity or identifying the risk factors [10]. Providing that the initial clinical diagnosis was correct, failure to address risk factors may lead to recurrence of the infection. In this regard, Gibson et al. [11] reported 10 patients who suffered from recalcitrant or recurrent oral candidiasis in whom diabetes mellitus was undiagnosed.

Chronic hyperplastic candidiasis typically presents as homogenous or speckled white lesion commonly on the buccal mucosa or lateral border of the tongue. It has a strong association with tobacco smoking [12] in addition to the other well-known risk factors. Complete resolution appears to be dependent on cessation of smoking in addition to the other therapeutic measures.

Some patients may have more than one predisposing factor simultaneously. Therefore, the whole set of predisposing factors should be considered in the workout for a patient with oral candidiasis. A common malpractice is that once a predisposing factor was identified the treating dentist may not follow up other factors which may lead to unsatisfactory treatment and persistence of the infection. Some predisposing factors are, however, still far from being controlled, for example, HIV infection, malignancies, and continuous use of immunosuppressing agents such as in organ transplant recipients or patients with autoimmune diseases. Treating oral candidiasis in these situations necessitates the use of systemic antifungal agents (fluconazole or clotrimazole), followed by prophylactic antifungal therapy [13].

3.2. Wrong Diagnosis. Oral candidiasis may be overlooked. Atrophic erythematous tongue associated with pain and burning sensation (atrophic glossitis) can be manifestations of hematinic or nutritional deficiency, such as vitamin B12, folic acid, or iron deficiencies [14], and sometimes can be treated as such. These signs and symptoms have also a high probability of being a Candida-induced lesion (i.e., acute erythematous candidiasis) [15]. In this case, complete resolution is not expected without institution of antifungal therapy, in addition to the management of the deficiency state.

On the other hand, some oral lesions wrongly diagnosed as oral candidiasis will be unsuccessfully treated with antifungal agents. Kiat-Amnuay and Bouquot [16] reported a case of oral frictional keratosis in a breast-fed infant (breast-feeding keratosis) which was misdiagnosed as thrush and hence was 
TABLE 1: Host factors predisposing for oral candidiasis.

\begin{tabular}{ll}
\hline Systemic factors & Local factors \\
\hline Hematinic deficiency & Removable dental appliances \\
Vitamin B12 & $\begin{array}{l}\text { Poor oral or denture hygiene } \\
\text { Ferritin }\end{array}$ \\
Folic acid & High carbohydrate diet \\
Immunosuppressive drugs & Broad spectrum antibiotics \\
Endocrine disease & Trauma \\
Diabetes mellitus & Heavy smoking \\
Hypothyroidism & \\
$\quad$ Hypoparathyroidism & \\
Blood dyscrasias or advanced \\
malignancy \\
Radiotherapy/chemotherapy \\
Reduced immune status, for \\
example, HIV infection \\
$\begin{array}{l}\text { Physiologic states: old } \\
\text { age/infancy/pregnancy }\end{array}$
\end{tabular}

unresponsive to repeated antifungal therapy. It is the experience of the authors that some dentists prescribe oral antifungal agents for the management of noncandidiasis lesions such as geographic tongue or recurrent aphthous stomatitis.

3.3. Incomplete Eradication of Candida Cells. Complete eradication of the causative Candida not only from the lesion but also from the reservoir of infection is essential part of the management. For example, the source of pathogens in Candida-associated angular cheilitis is commonly the inside of the mouth [8]. Hence fungus eradication from the clinical lesion by applying topical antifungal agents to the mouth angles only is inadequate management.

It has been proven that, in Candida-associated denture stomatitis, the fitting surface of the denture constitutes the reservoir of infection, where yeast cells are entrapped in the irregularities in denture-base or denture-relining materials [17]. Therefore, eradicating the yeast from the inflamed palatal mucosa without disinfecting the dentures will lead to recurrence of infection. In this regard, diet and other denturerelated factors should be taken into consideration such as good denture hygiene and refraining from day and night wearing of the dentures [18]. Unless the patient is educated about the denture hygiene and wearing and the proper fitting of denture is maintained, stomatitis will recur when antifungal therapy is discontinued [19].

\subsection{Insufficient Patient Instruction on Antifungal Therapy.} Nystatin and amphotericin B, the polyene antifungal agents which were first developed in the late 1950s, are still the mainstay for treating oral candidiasis. They are presented in different formulas such as pastilles, lozenges, suspension, troches, suppositories, and coated tablets. Lack of patient's instruction on the drug use may lead to suboptimal results. For example, nystatin and amphotericin B are not absorbed from the gastrointestinal tract if taken orally, but they act topically [20]. Swallowing the tablets or pastilles, rather than sucking or dissolving them in the mouth, is ineffective in treating oral candidiasis.

Topical antifungal agents have to be used regularly and for prolonged time to insure complete elimination of the fungus and resolution of the disease. It is widely clinically accepted rule that the patient has to use the nystatin or topical amphotericin B double the time needed for resolution of the clinical signs of infection. Due to taste intolerance to nystatin and amphotericin B and the relatively prolonged treatment period, patient's compliance with topical antifungal agents may be compromised [21]. Not instructing the patient on the treatment duration may lead to premature stop of the therapy and subsequent recurrence of the infection.

3.5. Candida Biofilm. Candida is present in the oral cavity in two distinct forms, as floating planktonic cells (blastopores, blastoconidia) and/or in an organized biofilm. Biofilm is defined as structured microbial community that is attached to a surface and surrounded by a self-produced extracellular matrix [22]. Biofilms are found adhering to living tissue such as mucosal surfaces or to abiotic surfaces such as implanted medical devices, intravascular catheters, and oral prostheses. Generally, C. albicans abiotic-surface biofilms are associated with increased drug resistance compared to planktonic cells [23]. The lower toxicity of clinically used antifungals, such as amphotericin B and fluconazole, to biofilm cells is due to extracellular matrix adsorption of drugs [24] and formation of "persister" cells [25]. Two components of the extracellular matrix, namely, $\beta$-glucan and extracellular DNA, promote biofilm resistance to multiple antifungals [26]. Dietary habits may influence resistance of fungi in biofilms to antifungal agents since biofilms on acrylic surfaces exposed to sugars showed higher Candida counts, phospholipase activity, and increased production of extracellular matrix substance (metabolic activity) [27]. In vivo [28] and ex vivo studies [29] have shown that planktonic Candida cells exhibit variable sensitivity to antifungal agents compared to those in biofilm. Although C. albicans is usually susceptible to all commonly used antifungals when tested in vitro, their biofilm form is highly resistant to most antifungals [30].

It is recommended that azole antifungals should be avoided for patients suffering from recurrent oral yeast infections due to a risk of selection and enrichment of resistant strains within the biofilm. On the contrary, lipid-formulation amphotericins and the echinocandins uniquely exhibit activity against mature biofilms [31].

Oropharyngeal candidiasis biofilm is more complex than biofilms on abiotic surfaces. The extracellular matrix layer of the former contains commensal bacterial flora and host components such as neutrophils and keratin from desquamating epithelial cells. Moreover, the extracellular matrix layer is abundant on cells at the basal end of biofilm close to the mucosal tissue and on cells invading the submucosal compartment; [32] hence long-term antifungal therapy is necessary.

3.6. Candida Resistance to Antifungal Agents. The recently witnessed increase in candidiases caused by non-albicans 
species, particularly C. glabrata and C. parapsilosis, is attributed mainly to the emergence of resistance following the widespread use of antifungal agents in prophylaxis and therapy [33]. This type of acquired resistance has been reported in C. albicans strains responsible for oral infections in HIV-positive patients undergoing repeated azole treatment. Nevertheless, some fungi, such as C. Krusei and C. glabrata, are genetically resistant to fluconazole [34], so more modern azole, such as itraconazole, is now in use for infections with these species. Echinocandin class of antifungal agents has recently emerged as an alternative to polyenes and azoles [35]. Therefore, accurate identification of the causative Candida species by culturing and sensitivity tests is important for the appropriate selection of the antifungal therapy.

\section{Conclusions}

Obtaining a thorough medical history and performing the appropriate workout for cases of oral candidiasis are mandatory for successful management. Treating dentist should be knowledgeable with action and indications and doses of antifungal agents. Certain predisposing factors are more difficult, if not impossible, to eradicate which necessitate the prophylactic antifungal therapy. In addition, patient education on the use of antifungal therapy is also essential.

\section{Disclosure}

Azmi M. G. Darwazeh is a Professor at the Department of Oral Medicine \& Surgery, Faculty of Dentistry, Jordan University of Science \& Technology. Tamer A. Darwazeh is a maxillofacial surgeon.

\section{Conflict of Interests}

The authors declare that there is no conflict of interests regarding the publication of this paper.

\section{References}

[1] L. P. Samaranayake, "Oral mycoses in HIV infection," Oral Surgery Oral Medicine and Oral Pathology, vol. 73, no. 2, pp. 171-180, 1992.

[2] F. Schauer and R. Hanschke, "Taxonomy and ecology of the genus Candida," Mycoses, vol. 42, no. S1, pp. 12-21, 1999.

[3] F. Gallè, M. Sanguinetti, G. Colella et al., "Oral candidosis: characterization of a sample of recurrent infections and study of resistance determinants," New Microbiologica, vol. 34, no. 4, pp. 379-389, 2011.

[4] J. A. Vazquez, "Invasive fungal infections in the intensive care unit," Seminars in Respiratory and Critical Care Medicine, vol. 31, no. 1, pp. 79-86, 2010.

[5] D. W. Williams, R. P. C. Jordan, X. Q. We et al., "Interactions of Candida albicans with host epithelial surfaces," Journal of Oral Microbiology, vol. 5, pp. 224-234, 2013.

[6] I. Olsen, "Oral adhesion of yeasts," Acta Odontologica Scandinavica, vol. 48, no. 1, pp. 45-53, 1990.

[7] S. W. Redding, M. C. Dahiya, W. R. Kirkpatrick et al., "Candida glabrata is an emerging cause of oropharyngeal candidiasis in patients receiving radiation for head and neck cancer," Oral Surgery, Oral Medicine, Oral Pathology, Oral Radiology, and Endodontics, vol. 97, no. 1, pp. 47-52, 2004.

[8] H. Egusa, N. S. Soysa, A. N. Ellepola, H. Yatani, and L. P. Samaranayake, "Oral candidosis in HIV-infected patients," Current HIV Research, vol. 6, no. 6, pp. 485-499, 2008.

[9] H. Terai and M. Shimahara, "Usefulness of culture test and direct examination for the diagnosis of oral atrophic candidiasis," International Journal of Dermatology, vol. 48, no. 4, pp. 371-373, 2009.

[10] R. Morgan, J. Tsang, N. Harrington, and L. Fook, "Survey of hospital doctors' attitudes and knowledge of oral conditions in older patients," Postgraduate Medical Journal, vol. 77, no. 908, pp. 392-394, 2001.

[11] J. Gibson, P.-J. Lamey, M. Lewis, and B. Frier, "Oral manifestations of previously undiagnosed non-insulin dependent diabetes mellitus," Journal of Oral Pathology and Medicine, vol. 19, no. 6, pp. 284-287, 1990.

[12] J. L. Rindum, A. Stenderup, and P. Holmstrup, "Identification of Candida albicans types related to healthy and pathological oral mucosa," Journal of Oral Pathology and Medicine, vol. 23, no. 9, pp. 406-412, 1994.

[13] G. R. Thompson III, P. K. Patel, W. R. Kirkpatrick et al., "Oropharyngeal candidiasis in the era of antiretroviral therapy," Oral Surgery, Oral Medicine, Oral Pathology, Oral Radiology and Endodontology, vol. 109, no. 4, pp. 488-495, 2010.

[14] A. Sun, H.-P. Lin, Y.-P. Wang, and C.-P. Chiang, "Significant association of deficiency of hemoglobin, iron and vitamin B12, high homocysteine level, and gastric parietal cell antibody positivity with atrophic glossitis," Journal of Oral Pathology \& Medicine, vol. 41, no. 6, pp. 500-504, 2012.

[15] H. Terai and M. Shimahara, "Atrophic tongue associated with Candida," Journal of Oral Pathology and Medicine, vol. 34, no. 7, pp. 397-400, 2005.

[16] S. Kiat-Amnuay and J. Bouquot, "Breastfeeding keratosis: this frictional keratosis of newborns may mimic thrush," Pediatrics, vol. 132, no. 3, pp. e775-e778, 2013.

[17] A. V. Abbeele, H. De Meel, M. Ahariz, J.-P. Perraudin, I. Beyer, and P. Courtois, "Denture contamination by yeasts in the elderly," Gerodontology, vol. 25, no. 4, pp. 222-228, 2008.

[18] Z. N. Al-Dwairi, "Prevalence and risk factors associated with denture-related stomatitis in healthy subjects attending a dental teaching hospital in North Jordan.", Journal of the Irish Dental Association, vol. 54, no. 2, pp. 80-83, 2008.

[19] L. Gendreau and Z. G. Loewy, "Epidemiology and etiology of denture stomatitis," Journal of Prosthodontics, vol. 20, no. 4, pp. 251-260, 2011.

[20] A. Y. Zhang, W. L. Camp, and B. E. Elewski, "Advances in topical and systemic antifungals," Dermatologic Clinics, vol. 25, no. 2, pp. 165-183, 2007.

[21] J. B. Epstein, E. L. Truelove, K. Hanson-Huggins et al., “Topical polyene antifungals in hematopoietic cell transplant patients: tolerability and efficacy," Supportive Care in Cancer, vol. 12, no. 7, pp. 517-525, 2004.

[22] J. W. Costerton, Z. Lewandowski, D. E. Caldwell, D. R. Korber, and H. M. Lappin-Scott, "Microbial biofilms," Annual Review of Microbiology, vol. 49, pp. 711-745, 1995.

[23] J. S. Finkel and A. P. Mitchell, "Genetic control of Candida albicans biofilm development," Nature Reviews Microbiology, vol. 9, no. 2, pp. 109-118, 2011. 
[24] J. Nett, L. Lincoln, K. Marchillo et al., "Putative role of $\beta-1,3$ glucans in Candida albicans biofilm resistance," Antimicrobial Agents and Chemotherapy, vol. 51, no. 2, pp. 510-520, 2007.

[25] K. Lewis, "Persister cells," Annual Review of Microbiology, vol. 64, pp. 357-372, 2010.

[26] H. T. Taff, K. F. Mitchell, J. A. Edward, and D. R. Andes, "Mechanisms of Candida biofilm drug resistance," Future Microbiology, vol. 8, no. 10, pp. 1325-1337, 2013.

[27] I. L. Santana, L. M. Gonçalves, A. A. D. Vasconcellos, W. J. da Silva, J. A. Cury, and A. A. D. B. Cury, "Dietary carbohydrates modulate Candida albicans biofilm development on the denture surface," PLoS ONE, vol. 8, no. 5, Article ID e64645, 2013.

[28] J. E. Nett, K. Marchillo, C. A. Spiegel, and D. R. Andes, "Development and validation of an in vivo Candida albicans biofilm denture model," Infection and Immunity, vol. 78, no. 9, pp. 3650-3659, 2010.

[29] J. Chandra, P. K. Mukherjee, S. D. Leidich et al., "Antifungal resistance of Candidal biofilms formed on denture acrylic in vitro," Journal of Dental Research, vol. 80, no. 3, pp. 903-908, 2001.

[30] R. Rautemaa and G. Ramage, "Oral candidosis-clinical challenges of a biofilm disease," Critical Reviews in Microbiology, vol. 37, no. 4, pp. 328-336, 2011.

[31] D. M. Kuhn and M. A. Ghannoum, "Candida biofilms: antifungal resistance and emerging therapeutic options," Current Opinion in Investigational Drugs, vol. 5, no. 2, pp. 186-197, 2004.

[32] S. Ganguly and A. P. Mitchell, "Mechanisms of Candida biofilm drug resistance. Mucosal biofilms of Candida albicans," Current Opinion in Microbiology, vol. 14, pp. 380-385, 2011.

[33] M. Ruhnke, "Epidemiology of Candida albicans infections and role of non-Candida-albicans yeasts," Current Drug Targets, vol. 7, no. 4, pp. 495-504, 2006.

[34] Y.-L. Yang and H.-J. Lo, "Mechanisms of antifungal agent resistance," Journal of Microbiology, Immunology and Infection, vol. 34, no. 2, pp. 79-86, 2001.

[35] A. M. Bal, “The echinocandins: three useful choices or three too many?" International Journal of Antimicrobial Agents, vol. 35, no. 1, pp. 13-18, 2010. 

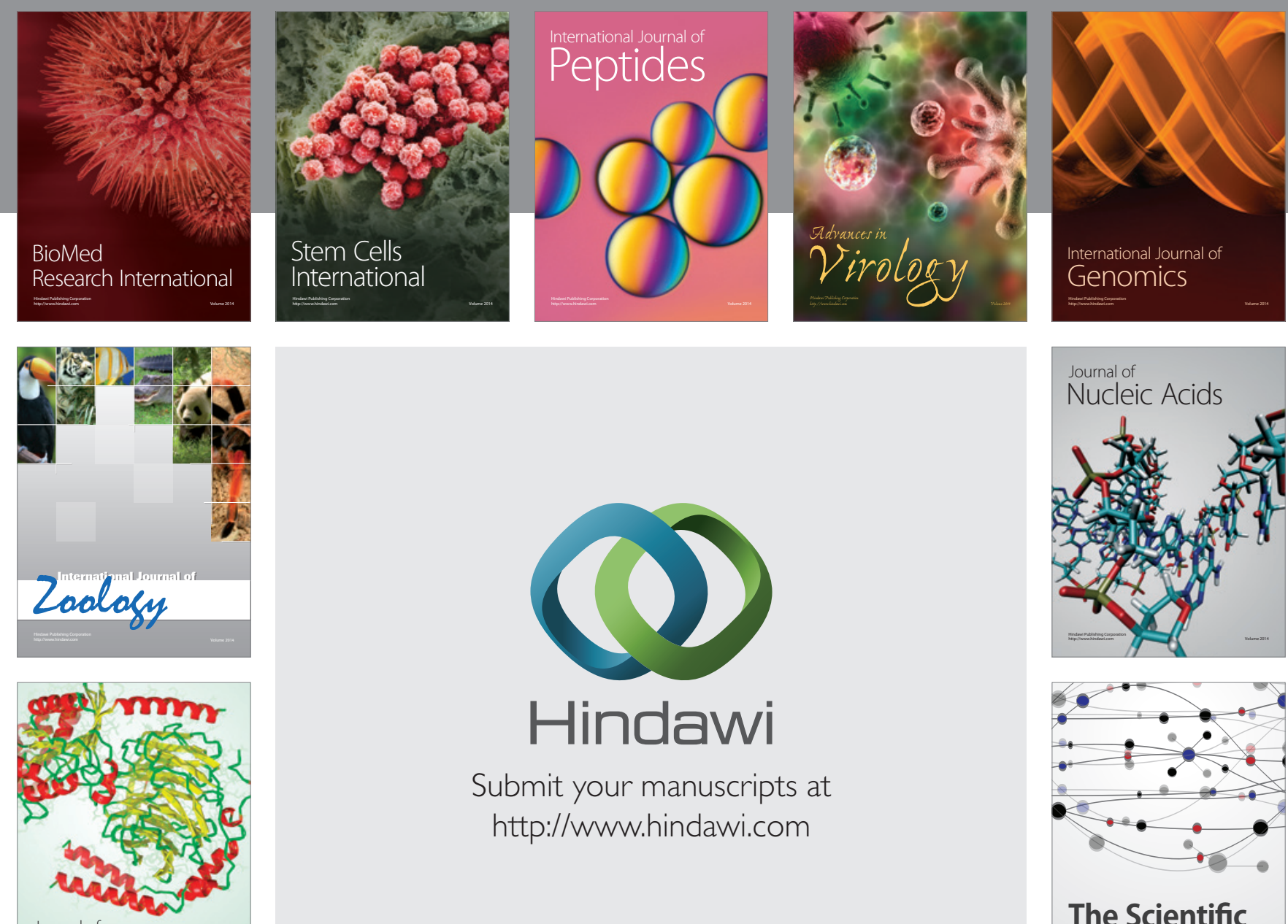

Submit your manuscripts at

http://www.hindawi.com

Journal of
Signal Transduction
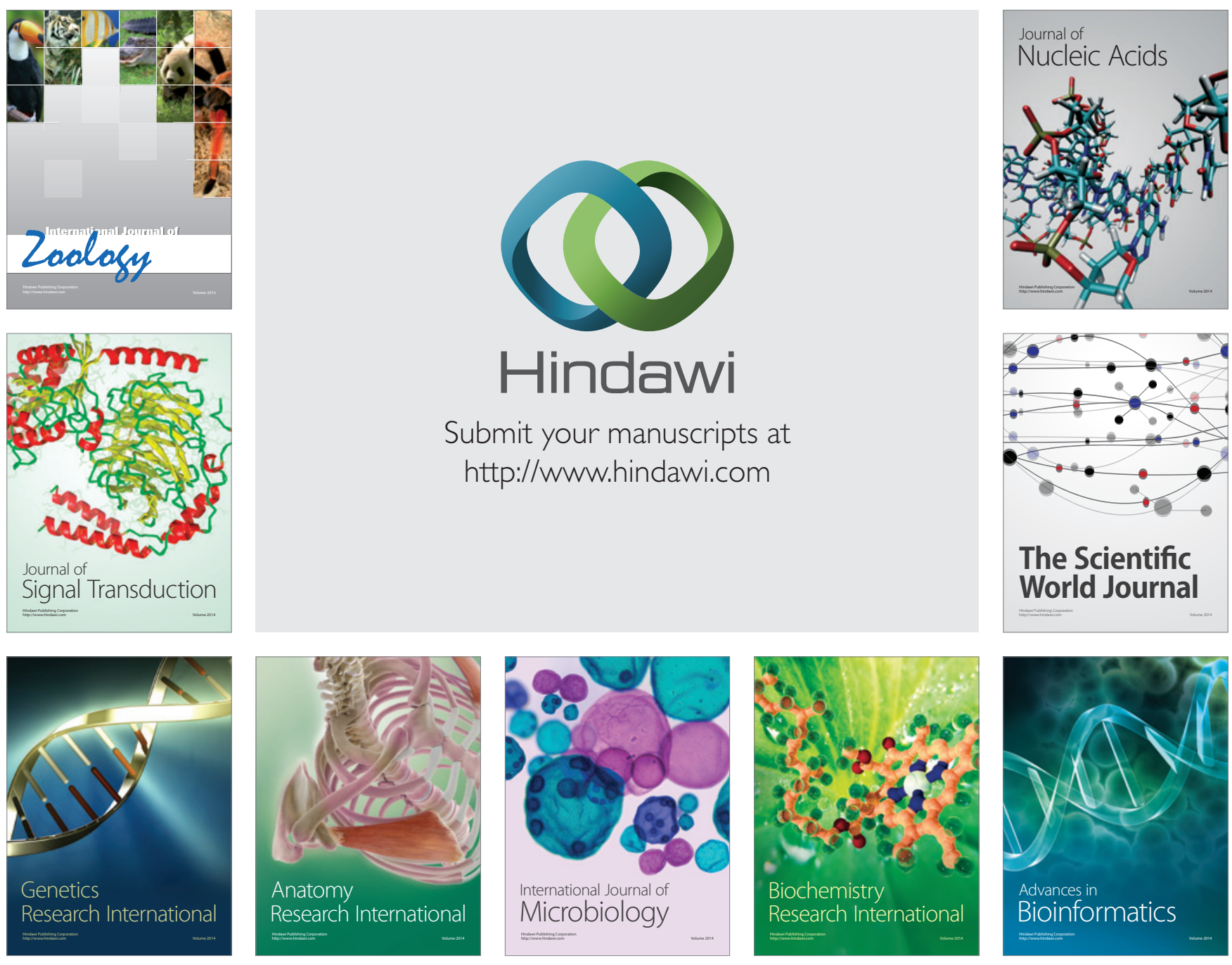

The Scientific World Journal
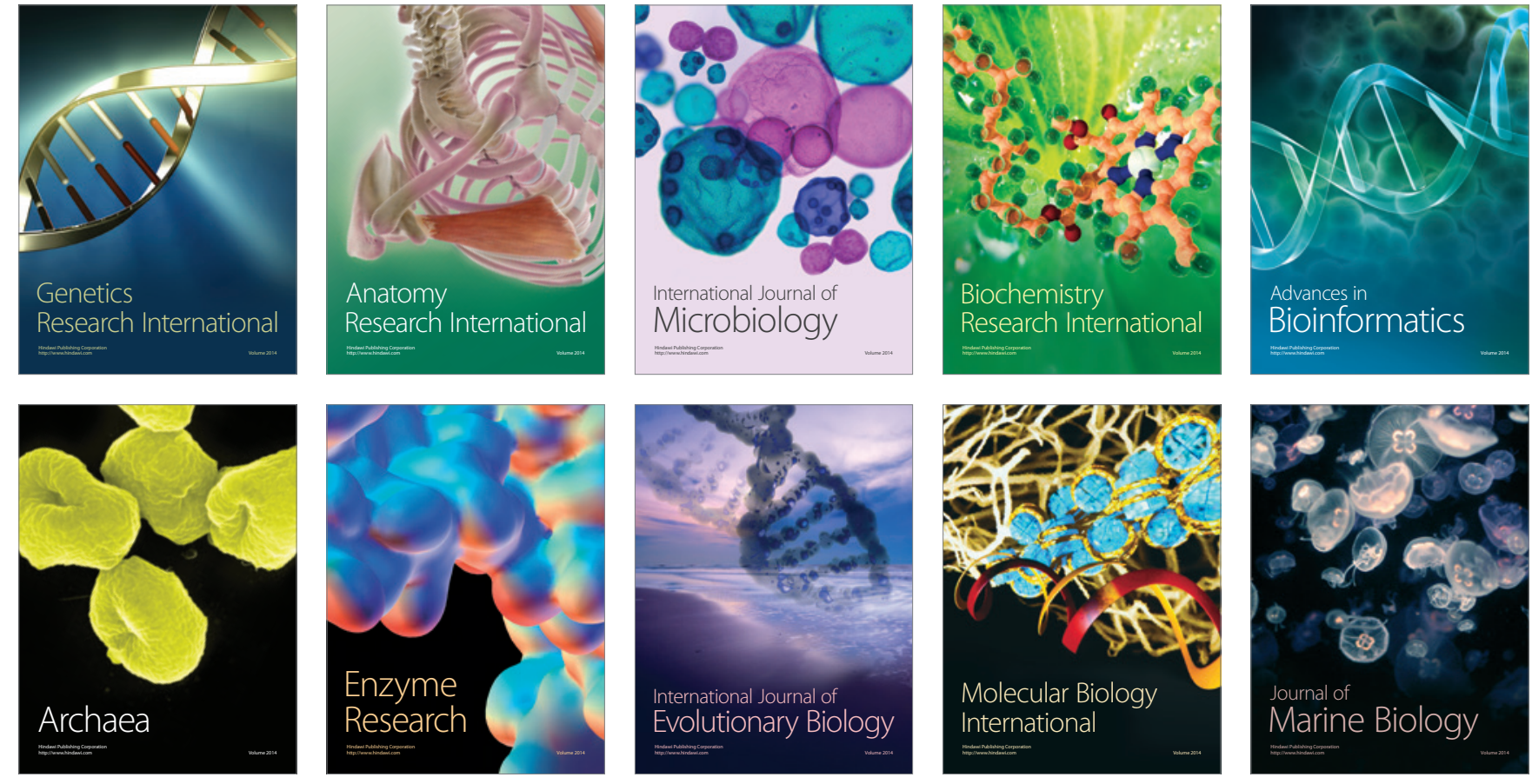